\title{
E-cigarette use behaviors and device characteristics of daily exclusive e-cigarette users in Maryland: Implications for product toxicity
}

\author{
Angela Aherrera ${ }^{1,2 *}$, Atul Aravindakshan ${ }^{1 *}$, Stephanie Jarmul 1,3, Pablo Olmedo ${ }^{1,4,5}$, Rui Chen ${ }^{1}$, Joanna E. Cohen ${ }^{6}$, Ana \\ Navas-Acien ${ }^{1,4}$, Ana M. Rule
}

\begin{abstract}
INTRODUCTION Few studies to date have characterized daily exclusive e-cigarette users, device characteristics, and use behaviors. This study describes daily e-cigarette user characteristics, and assesses the association between user behaviors and demographics.

METHODS From 2015-2017, 100 daily exclusive e-cigarette users and 50 nonusers were recruited in Maryland, USA. Sociodemographic characteristics, health status, e-cigarette/tobacco use behaviors, device characteristics, and reasons for e-cigarette use were collected by interview. Chi-squared tests (categorical variables), Student's t-test (continuous variables), and linear regressions were used to assess relationships between variables. RESULTS Most daily exclusive e-cigarette users were men, White, former smokers, used MODs/tanks, and vaped on average 365 puffs/day (SD: 720). A third of users first vaped within 5 minutes of waking in the morning, and $56 \%$ vaped throughout the day. E-liquid consumption ranged from 5-240 mL/week (median: 32.5 ), with nicotine concentration 0-24 mg/mL (median: 3). E-cigarette users were more likely to report wheezing/whistling and hypertension than controls, although the finding was not statistically significant after adjustment. Less than half planned to quit vaping.

CONCLUSIONS Daily e-cigarette users between 2015-2017 most commonly vaped MOD/tank devices. Being male and of lower education was associated with higher usage. Daily users with no intention to quit may be at risk for increased exposure to emissions from e-cigarettes that include inorganic (metals) and organic (e.g. acrolein, formaldehyde) compounds with known toxic effects, particularly to the lung. Further research is needed to characterize the long-term health effects of daily e-cigarette use.
\end{abstract}

\author{
AFFILIATION \\ 1 Department of Environmental \\ Health and Engineering, Johns Hopkins \\ Bloomberg School of Public Health, \\ Baltimore, United States \\ 2 Division of Pediatric Pulmonary \\ Medicine, Johns Hopkins School of \\ Medicine, Baltimore, United States \\ 3 Department of Population Health, \\ London School of Hygiene and Tropical \\ Medicine, London, United Kingdom \\ 4 Department of Environmental Health \\ Sciences, Columbia University, New York, \\ United States \\ 5 Department of Legal Medicine, \\ Toxicology and Physical Anthropology, \\ Faculty of Medicine, University of \\ Granada, Granada, Spain \\ 6 Department of Health, Behavior and \\ Society, Johns Hopkins Bloomberg School \\ of Public Health, Baltimore, United States \\ \#Contributed equally, co-first authors
}

\section{CORRESPONDENCE TO}

Angela Aherrera. Department of Environmental Health and Engineering,

Johns Hopkins Bloomberg School of

Public Health, 615 N. Wolfe St., Office

E6640, Baltimore, MD 21205, United

States.

E-mail: aaherre2@jhu.edu

\section{KEYWORDS}

nicotine, health implications,

e-cigarettes, device characteristics, use

patterns and behaviors

Received: 20 May 2020

Revised: 13 August 2020

Accepted: 9 October 2020

\section{INTRODUCTION}

The use of electronic cigarettes (e-cigarettes) has significantly increased over the past decade, with rapid changes in demographic characteristics as new devices are introduced in the market. The number of current e-cigarette users in middle and high school in the US increased from 2.1 million in 2017 to 3.6 million in $2018^{1}$. E-cigarettes are composed 
of a battery, a cartridge containing e-liquid, and an atomizer, which heats and aerosolizes the e-liquid using a metal coil. E-cigarette devices have rapidly evolved in the last 10 years. First generation devices are closed, non-refillable (disposable) systems ${ }^{2}$ called 'cig-a-likes', which resemble a cigarette and consist of a cartridge (called cartomizer) that contains the e-liquid in contact with the coil, and a low-capacity rechargeable battery. The next generation devices include e-pen models (2nd generation) and tank-like systems (3rd generation), which are open, refillable, reusable systems, and were common among former smokers between 2015 and $2017^{3}$. Third generation open devices (called modifiable e-cigarettes or MODs) are typically larger, with a more powerful battery and adjustable voltage/wattage delivery, a refillable e-liquid reservoir, and replaceable heating coils. The fourth generation devices, called PODs, commonly used by new e-cigarette users and youth, resemble the first generation closed systems, with a low capacity battery, and a disposable 'pod' that contains high concentration nicotine-laden liquid in contact with a coil ${ }^{4}$. As of 2020 , the newest generation devices include hybrid low-battery devices with refillable pods, and completely disposable devices ${ }^{5}$. In all generations, the heating coils used to generate the aerosol are typically made up of metal alloys. Commonly used coils include Kanthal (chromium, aluminum, iron), Nichrome (nickel and chromium), and stainless steel (nickel, chromium, carbon) ${ }^{6}$.

Few studies have characterized daily e-cigarette users, their devices and use behaviors, and their perceptions of e-cigarette safety. Daily e-cigarette users represent a small subgroup (19\%) of the e-cigarette population compared to intermittent (29\%) and occasional (51\%) e-cigarette users, but they are particularly at risk of any potential long-term consequences of e-cigarette use, given the intensity of $u^{7} e^{7}$. Moreover, while nationally representative studies such as the Population Assessment of Tobacco and Health (PATH) study and the National Health Interview Survey (NHIS) have begun including questions regarding e-cigarette use, they are limited in asking questions pertaining to e-cigarette device characteristics (including voltage, power, and the type of heating coil used) and use behaviors (including the amount of e-liquid consumed per week, the number of times the heating coil is replaced per month, and the number of puffs taken per day). Describing daily use is critical in understanding the chronic exposure that could potentially result in long-term health effects.

The purpose of this study was to evaluate demographic characteristics, e-cigarette use behaviors, reasons for use, and self-reported health status of daily exclusive e-cigarette users, and to compare with matched non-users (those who neither vape e-cigarettes nor smoke combustible cigarettes). We describe e-cigarette device characteristics, vaping frequency, and e-liquid nicotine concentrations in association with user demographics among e-cigarette users in Maryland to better identify the types of users at risk and to understand the practices that may influence the potential toxicity of e-cigarettes among daily users.

\section{METHODS}

\section{Study population and recruitment}

E-cigarette users were recruited through advertisements and flyers posted in universities, local newspapers and online advertisement websites (City Paper, Craigslist), social media platforms (Facebook), and e-cigarette (vape) shops and conventions between December 2015 and October 2017 in Maryland, USA. Participants were residents of Maryland, aged $\geq 18$ years, and not pregnant at the time of recruitment. The goal was to recruit 50 daily exclusive e-cigarette users during the first wave of recruitment (December 2015 to March 2016), and 50 daily exclusive e-cigarette users and 50 non-users during the second wave (March 2017 to October 2017). Exclusive e-cigarette users were defined as non-cigarette smokers or former smokers who had quit at least 6 months before enrollment and vaped daily for at least 6 weeks. Users were instructed to bring their e-cigarette devices to the study, which could either be an open or closed system. It should be noted that at the time of recruitment, none of the participants were POD users. Non-users were defined as non-cigarette smokers and non-e-cigarette users, or former cigarette smokers who quit at least 6 months prior to enrollment. To aid in the comparability, nonusers were matched to e-cigarette users according to age (within 5 years), sex, and race. The study protocol was approved by the Institutional Review Board at Johns Hopkins University (Baltimore, Maryland). All participants provided written informed consent. 


\section{Data collection}

After confirming eligibility, e-cigarette user participants were asked to carry out their normal vaping routine and bring their e-cigarette device to the study visit, which took place at the Johns Hopkins Bloomberg School of Public Health in Baltimore, MD. At the time of their appointment, participants responded to an interviewer-based questionnaire addressing sociodemographic characteristics, previous tobacco use, current e-cigarette use (including e-liquid consumed/week, preferred voltage, e-liquid nicotine concentrations), overall health status, beliefs/perceptions on e-cigarette safety, and indoor vaping and smoking rules to account for potential secondary exposure. Additional questions on e-cigarette use (including the number of puffs/day, average seconds/puff, days since last coil change) were added in the second year of recruitment. Intensity of nicotine addiction was assessed by adapting the Fagerström test for nicotine dependence ${ }^{8}$, while sensory and respiratory symptoms were addressed using a questionnaire commonly used in studies regarding tobacco smoking and exposure to tobacco smoke ${ }^{9}$.

\section{Statistical analysis}

We compared e-cigarette users and non-users by demographic characteristics, imposed house rules put in place about smoking and vaping indoors, and health characteristics using chi-squared tests for categorical variables and Student's t-test for continuous variables. We also compared male and female e-cigarette users by primary reasons for vaping, their intention to reduce nicotine, and intention to quit vaping using chi-squared tests. Lastly, we conducted linear regression models to analyze the association of age, sex, education, race, and previous smoking status, with preferred voltage, preferred nicotine concentration, e-liquid consumed/week, puff count/day, and seconds/puff before and after adjusting for those same indicators. Statistical analyses were conducted in Stata 14 (Stata Corp, College Station, TX). The level of statistical significance was set at $\mathrm{a}=0.05$.

\section{RESULTS}

\section{Participant characteristics}

One hundred and fifty participants (100 e-cigarette users and 50 non-users) were recruited (Table 1). Their mean age was 30.1 years (SD: 9.6), 64\% were men, and $83 \%$ were White. Compared to e-cigarette users, most non-users had a higher level of education $(90 \%)$ and were never smokers (90\%). Eighty-nine per cent of e-cigarette users were former smokers (compared to the $10 \%$ of non-users who were former smokers); they had an earlier age to first smoke cigarettes, and smoked more cigarettes per day before quitting (mean: 17 cigarettes/day; range: 1-80 cigarettes/day) (Table 1).

Table 1. Participant characteristics by vaping category $(\mathrm{N}=150)^{a}$

\begin{tabular}{|c|c|c|c|c|c|}
\hline General characteristics & $\mathbf{N}$ & Total & $\begin{array}{c}\text { E-cigarette } \\
\text { users }(n=100)\end{array}$ & $\begin{array}{l}\text { Non-users } \\
\quad(n=50)\end{array}$ & $p^{*}$ \\
\hline Age (years), mean (SD) & 150 & $30.1(9.6)$ & $30.3(9.2)$ & $29.7(10.5)$ & 0.70 \\
\hline \multicolumn{6}{|l|}{ Gender, \% } \\
\hline Male & 91 & 64.0 & 67.0 & 60.0 & \multirow{2}{*}{0.60} \\
\hline Female & 59 & 36.0 & 33.0 & 40.0 & \\
\hline \multicolumn{6}{|l|}{ Education level, \% } \\
\hline$\leq$ High school & 46 & 30.7 & 41.0 & 10.0 & \multirow{2}{*}{$<0.001$} \\
\hline >High school & 104 & 69.3 & 59.0 & 90.0 & \\
\hline \multicolumn{6}{|l|}{ Race, $\%$} \\
\hline White & 124 & 82.7 & 87.0 & 74.0 & \multirow{2}{*}{0.05} \\
\hline Non-White & 26 & 17.3 & 13.0 & 26.0 & \\
\hline \multicolumn{6}{|l|}{ Employed, \% } \\
\hline Yes & 99 & 66.0 & 75.0 & 48.0 & \multirow{3}{*}{0.001} \\
\hline No & 51 & 34.0 & 25.0 & 52.0 & \\
\hline Student & 29 & 19.3 & 9.00 & 40.0 & \\
\hline
\end{tabular}


Table 1. Continued

\begin{tabular}{|c|c|c|c|c|c|}
\hline General characteristics & $\mathbf{N}$ & Total & $\begin{array}{c}\text { E-cigarette } \\
\text { users }(n=100)\end{array}$ & $\begin{array}{l}\text { Non-users } \\
(n=50)\end{array}$ & $p^{*}$ \\
\hline \multicolumn{6}{|l|}{ Smoking status } \\
\hline Ever smoker, \% & 94 & 62.7 & 89.0 & 10.0 & \multirow{4}{*}{$<0.001$} \\
\hline Age (years), mean (SD) & & $31.2(9.4)$ & $30.3(9.2)$ & $31.6(12.0)$ & \\
\hline Never smoker, $\%$ & 56 & 37.3 & 11.0 & 90.0 & \\
\hline Age (years), mean (SD) & & $28.2(9.6)$ & $30.3(8.7)$ & $29.7(10.6)$ & \\
\hline $\begin{array}{l}\text { Age first smoked tobacco cigarettes } \\
\text { (years), mean (SD) }\end{array}$ & 94 & $15.4(2.9)$ & $15.1(2.5)$ & $19.8(5.7)$ & $<0.001$ \\
\hline $\begin{array}{l}\text { Time since quitting cigarettes (months), } \\
\text { mean (SD) }\end{array}$ & 91 & $23.7(18.2)$ & $23.2(18.1)$ & $33.5(19.8)$ & 0.27 \\
\hline $\begin{array}{l}\text { Cigarettes smoked daily before quitting, } \\
\text { mean (SD) }\end{array}$ & 92 & $16.3(11.9)$ & $16.8(11.9)$ & $4.5(3.8)$ & 0.04 \\
\hline Age to first vape (years), mean (SD) & 99 & & $28.3(9.9)$ & - & - \\
\hline
\end{tabular}

* Comparing exclusive e-cigarette users vs non-users. a Participants were recruited from Maryland from December 2015 - 0ctober 2017.

Table 2. Self-reported e-cigarette use behaviors and patterns $(\mathbf{N}=150)^{a}$

\begin{tabular}{|c|c|c|}
\hline E-cigarette use behaviors & $\mathbf{N}$ & $\begin{array}{c}E \text {-cigarette } \\
\text { users } \\
(n=100)\end{array}$ \\
\hline \multicolumn{3}{|l|}{ Time to first vape (min), $\%$} \\
\hline$<5$ & 27 & 27.0 \\
\hline $6-15$ & 9 & 9.0 \\
\hline $16-30$ & 29 & 29.0 \\
\hline $31-60$ & 24 & 24.0 \\
\hline$>60$ & 11 & 11.0 \\
\hline \multicolumn{3}{|l|}{ Number of different devices used ${ }^{a}, \%$} \\
\hline 1 & 45 & 46.0 \\
\hline 2 & 25 & 26.0 \\
\hline 3 & 13 & 13.0 \\
\hline 4 & 15 & 15.0 \\
\hline Number of puffs/day ${ }^{b}$, mean (SD) & 50 & $365.1(720.0)$ \\
\hline \multicolumn{3}{|l|}{ Portion of the day to vape ${ }^{b}, \%$} \\
\hline Morning & 4 & 8.0 \\
\hline Afternoon & 6 & 12.0 \\
\hline Evening & 12 & 24.0 \\
\hline Most of the day & 28 & 56.0 \\
\hline Average seconds/puff ${ }^{\mathrm{b}}$, mean (SD) & 50 & $4.0(2.0)$ \\
\hline \multicolumn{3}{|l|}{ E-liquid purchase location, $\%$} \\
\hline Vape shop & 77 & 79.0 \\
\hline Online & 14 & 14.0 \\
\hline Other & 6 & 6.0 \\
\hline $\begin{array}{l}\text { Preferred nicotine concentration (mg/ } \\
\mathrm{mL}), \text { median (range) }\end{array}$ & 98 & $3(0-24)$ \\
\hline $\begin{array}{l}\text { E-liquid consumed per week }(\mathrm{mL}) \text {, mean } \\
\text { (SD) }\end{array}$ & 98 & $53.3(48.4)$ \\
\hline
\end{tabular}

Table 2. Continued

\begin{tabular}{|c|c|c|}
\hline E-cigarette use behaviors & $\mathbf{N}$ & $\begin{array}{c}E \text {-cigarette } \\
\text { users } \\
(n=100)\end{array}$ \\
\hline Device power (W), mean (SD) & 96 & $56.3(30.8)$ \\
\hline Device voltage (V), mean (SD) & 92 & $4.21(1.2)$ \\
\hline \multicolumn{3}{|l|}{ Change voltage, $\%$} \\
\hline Yes & 85 & 87.0 \\
\hline No & 13 & 13.0 \\
\hline $\begin{array}{l}\text { How often change coil/month, mean } \\
\text { (SD) }\end{array}$ & 96 & $2.5(2.4)$ \\
\hline $\begin{array}{l}\text { Last time of coil change }(\text { days })^{b} \text {, mean } \\
\text { (SD) }\end{array}$ & 50 & $15.9(19.4)$ \\
\hline \multicolumn{3}{|l|}{ Knowledge of coil composition, $\%$} \\
\hline Yes & 87 & 87.0 \\
\hline No & 13 & 13.0 \\
\hline \multicolumn{3}{|l|}{ Type of coil used, \% } \\
\hline Kanthal & 44 & 50.6 \\
\hline Nichrome & 13 & 14.9 \\
\hline Pure nickel & 3 & 3.4 \\
\hline Stainless steel & 15 & 17.2 \\
\hline Titanium & 4 & 4.6 \\
\hline Combination with Kanthal & 8 & 9.2 \\
\hline
\end{tabular}

a Participants were recruited from Maryland from December 2015 - October 2017. b Year 2 data only.

\section{E-cigarette use patterns, device characteristics, and reasons for vaping}

Among e-cigarette users, the mean (SD) age at first vape was 28.3 (9.9) years (Table 1). By device 


\section{Research Paper}

Table 3. Mean difference (95\% CI) in e-cigarette use patterns by demographic characteristics analyzed using linear regression (N=150) a

\begin{tabular}{|c|c|c|c|c|c|c|c|c|c|c|c|c|c|c|c|}
\hline \multirow[t]{2}{*}{ Characteristic } & \multicolumn{3}{|c|}{$\begin{array}{l}\text { Voltage } \\
\text { (V) }\end{array}$} & \multicolumn{3}{|c|}{$\begin{array}{l}\text { Nicotine use } \\
(\mathrm{mg} / \mathrm{mL})\end{array}$} & \multicolumn{3}{|c|}{$\begin{array}{c}\text { E-liquid/week } \\
\text { (mL) }\end{array}$} & \multicolumn{3}{|c|}{ Puff count/day b } & \multicolumn{3}{|c|}{ Seconds/puff ${ }^{b}$} \\
\hline & $\mathbf{N}$ & $\begin{array}{c}\text { OR } \\
(95 \% \text { CI })\end{array}$ & $\begin{array}{c}\text { AOR } \\
(95 \% \mathrm{CI})\end{array}$ & $\mathbf{N}$ & $\begin{array}{c}\text { OR } \\
(95 \% \mathrm{CI})\end{array}$ & $\begin{array}{c}\text { AOR } \\
(95 \% \text { CI })\end{array}$ & $\mathbf{N}$ & $\begin{array}{c}\text { OR } \\
(95 \% \mathrm{CI})\end{array}$ & $\begin{array}{c}\text { AOR } \\
(95 \% \text { CI })\end{array}$ & $\mathbf{N}$ & $\begin{array}{c}\text { OR } \\
(95 \% \text { CI })\end{array}$ & $\begin{array}{c}\text { AOR } \\
(95 \% \text { CI })\end{array}$ & $\mathbf{N}$ & $\begin{array}{c}\text { OR } \\
(95 \% \text { CI })\end{array}$ & $\begin{array}{c}\text { AOR } \\
(95 \% \mathrm{CI})\end{array}$ \\
\hline Age (per year) & 92 & $\begin{array}{c}-0.01 \\
(-0.04,0.02)\end{array}$ & $\begin{array}{c}-0.01 \\
(-0.03,0.02)\end{array}$ & 98 & $\begin{array}{c}0.24 \\
(0.13,0.34)\end{array}$ & $\begin{array}{c}0.24 \\
(0.12,0.36)\end{array}$ & 98 & $\begin{array}{c}-0.04 \\
(-1.10,1.02)\end{array}$ & $\begin{array}{c}-0.46 \\
(-1.55,0.63)\end{array}$ & 50 & $\begin{array}{c}-20.3 \\
(-43.0,2.39)\end{array}$ & $\begin{array}{c}-25.1 \\
(-49.9,-0.25)\end{array}$ & 50 & $\begin{array}{c}0.01 \\
(-0.05,0.08)\end{array}$ & $\begin{array}{c}0.02 \\
(-0.06,0.09)\end{array}$ \\
\hline \multicolumn{16}{|l|}{ Gender } \\
\hline Male & 65 & $\begin{array}{l}0.00 \\
\text { (Ref.) }\end{array}$ & $\begin{array}{l}0.00 \\
\text { (Ref.) }\end{array}$ & 66 & $\begin{array}{l}0.00 \\
\text { (Ref.) }\end{array}$ & $\begin{array}{c}0.00 \\
\text { (Ref.) }\end{array}$ & 67 & $\begin{array}{l}0.00 \\
\text { (Ref.) }\end{array}$ & $\begin{array}{c}0.00 \\
\text { (Ref.) }\end{array}$ & 35 & $\begin{array}{l}0.00 \\
\text { (Ref.) }\end{array}$ & $\begin{array}{l}0.00 \\
\text { (Ref.) }\end{array}$ & 35 & $\begin{array}{c}0.00 \\
\text { (Ref.) }\end{array}$ & $\begin{array}{c}0.00 \\
\text { (Ref.) }\end{array}$ \\
\hline Female & 27 & $\begin{array}{c}-0.46 \\
(-1.00,0.07)\end{array}$ & $\begin{array}{c}-0.54 \\
(-1.04,-0.03)\end{array}$ & 32 & $\begin{array}{c}0.04 \\
(-2.24,2.33)\end{array}$ & $\begin{array}{c}0.20 \\
(-1.95,2.34)\end{array}$ & 31 & $\begin{array}{c}-23.5 \\
(-43.9,-3.11)\end{array}$ & $\begin{array}{c}-22.7 \\
(-42.9,-2.46)\end{array}$ & 15 & $\begin{array}{c}-103.4 \\
(-553,347)\end{array}$ & $\begin{array}{c}-132.2 \\
(-49.9,-0.25)\end{array}$ & 15 & $\begin{array}{c}0.34 \\
(-0.88,1.57)\end{array}$ & $\begin{array}{c}0.40 \\
(-0.92,1.73)\end{array}$ \\
\hline \multicolumn{16}{|l|}{ Education } \\
\hline$\leq \mathrm{HS}$ & 38 & $\begin{array}{c}0.00 \\
\text { (Ref.) }\end{array}$ & $\begin{array}{c}0.00 \\
\text { (Ref.) }\end{array}$ & 41 & $\begin{array}{c}0.00 \\
\text { (Ref.) }\end{array}$ & $\begin{array}{c}0.00 \\
\text { (Ref.) }\end{array}$ & 41 & $\begin{array}{c}0.00 \\
\text { (Ref.) }\end{array}$ & $\begin{array}{c}0.00 \\
\text { (Ref.) }\end{array}$ & 19 & $\begin{array}{c}0.00 \\
\text { (Ref.) }\end{array}$ & $\begin{array}{c}0.00 \\
\text { (Ref.) }\end{array}$ & 19 & $\begin{array}{c}0.00 \\
\text { (Ref.) }\end{array}$ & $\begin{array}{c}0.00 \\
\text { (Ref.) }\end{array}$ \\
\hline$>\mathrm{HS}$ & 54 & $\begin{array}{c}-0.13 \\
(-0.63,0.37)\end{array}$ & $\begin{array}{c}-0.12 \\
(-0.60,0.36)\end{array}$ & 57 & $\begin{array}{c}-0.90 \\
(-3.07,1.27)\end{array}$ & $\begin{array}{c}-0.02 \\
(-2.09,2.04)\end{array}$ & 57 & $\begin{array}{c}-20.3 \\
(-39.6,-0.93)\end{array}$ & $\begin{array}{c}-20.4 \\
(-39.7,-1.09)\end{array}$ & 31 & $\begin{array}{c}202.8 \\
(-219,625)\end{array}$ & $\begin{array}{c}134.1 \\
(-301,570)\end{array}$ & 31 & $\begin{array}{c}-0.23 \\
(-1.38,0.93)\end{array}$ & $\begin{array}{c}-0.25 \\
(-1.50,1.01)\end{array}$ \\
\hline \multicolumn{16}{|l|}{ Race } \\
\hline Non-White & 8 & $\begin{array}{c}-0.13 \\
(-1.00,0.75)\end{array}$ & $\begin{array}{c}-0.19 \\
(-1.03,0.65)\end{array}$ & 12 & $\begin{array}{c}-2.22 \\
(-5.47,1.01)\end{array}$ & $\begin{array}{c}-1.12 \\
(-4.22,1.98)\end{array}$ & 11 & $\begin{array}{c}-25.0 \\
(-55.5,5.48)\end{array}$ & $\begin{array}{c}-25.5 \\
(-55.8,4.73)\end{array}$ & 7 & $\begin{array}{c}-245 \\
(-837,346)\end{array}$ & $\begin{array}{c}-342 \\
(-968,284)\end{array}$ & 7 & $\begin{array}{c}0.09 \\
(-1.53,1.71)\end{array}$ & $\begin{array}{c}0.07 \\
(-1.74,1.87)\end{array}$ \\
\hline \multicolumn{16}{|c|}{ Previous smoker } \\
\hline No & 10 & $\begin{array}{l}0.00 \\
\text { (Ref.) }\end{array}$ & $\begin{array}{l}0.00 \\
\text { (Ref.) }\end{array}$ & 11 & $\begin{array}{l}0.00 \\
\text { (Ref.) }\end{array}$ & $\begin{array}{c}0.00 \\
\text { (Ref.) }\end{array}$ & 11 & $\begin{array}{c}0.00 \\
\text { (Ref.) }\end{array}$ & $\begin{array}{c}0.00 \\
\text { (Ref.) }\end{array}$ & 6 & $\begin{array}{c}0.00 \\
\text { (Ref.) }\end{array}$ & $\begin{array}{l}0.00 \\
\text { (Ref.) }\end{array}$ & 6 & $\begin{array}{c}0.00 \\
\text { (Ref.) }\end{array}$ & $\begin{array}{c}0.00 \\
\text { (Ref.) }\end{array}$ \\
\hline Yes & 82 & $\begin{array}{c}-1.28 \\
(-2.03,-0.54)\end{array}$ & $\begin{array}{c}-1.31 \\
(-2.10,-0.53)\end{array}$ & 87 & $\begin{array}{c}0.75 \\
(-2.64,4.14)\end{array}$ & $\begin{array}{c}-1.09 \\
(-4.38,2.19)\end{array}$ & 87 & $\begin{array}{c}0.17 \\
(-30.7,31.1)\end{array}$ & $\begin{array}{c}-3.10 \\
(-33.9,27.7)\end{array}$ & 44 & $\begin{array}{c}39.5 \\
(-597,676)\end{array}$ & $\begin{array}{c}247 \\
(-423,919)\end{array}$ & 44 & $\begin{array}{c}-1.04 \\
(-1.84,1.63)\end{array}$ & $\begin{array}{c}-0.14 \\
(-2.08,1.79)\end{array}$ \\
\hline
\end{tabular}

a Participants were recruited from Maryland from December 2015 - October 2017. b Only year 2 data. OR: odds ratio. AOR: adjusted odds ratio, adjusted for age, gender, education level, race, and previous smoking status. HS: High school. 
type, only 2 participants used 1st generation devices while 98 users used 2 nd or 3 rd generation devices. More than a third (36\%) of users first vaped within 15 minutes of waking in the morning, with $27 \%$ vaping within 5 minutes (Table 2). Over half of participants owned two or more devices (54\%), and vaped continuously throughout the day (56\%). Most users $(87 \%)$ were knowledgeable about the coil, with Kanthal or some combination with Kanthal (51\%), stainless steel (17\%), and Nichrome (15\%) being the most commonly used coils. Users' coils were last changed at an average of 16 (SD: 19) days before coming to the study session, and replaced at an average of 3 (SD: 2 ) times per month. The reported mean voltage was $4.21 \mathrm{~V}$ (range: $2.12-12.50 \mathrm{~V}$ ), and $85 \%$ reported periodically changing the voltage of the device. Men used a higher voltage than women, and former smokers used a lower voltage than never smokers (Table 3). According to e-liquid characteristics and use, $79 \%$ of the study population purchased their e-liquid from a vape shop, $14 \%$ online, and the remaining $7 \%$ from other sources such as making their own or receiving it from a friend. E-liquid consumption varied greatly, ranging from

Table 4. Primary reasons for vaping and intention to quit $(\mathrm{N}=150)^{\text {a }}$

\begin{tabular}{|c|c|c|c|c|c|}
\hline Characteristic & $\mathbf{N}$ & $\begin{array}{c}\text { Total } \\
\%\end{array}$ & $\begin{array}{c}\text { Men } \\
\%\end{array}$ & $\begin{array}{l}\text { Women } \\
\%\end{array}$ & $p$ \\
\hline $\begin{array}{l}\text { Primary reasons for } \\
\text { vaping }\end{array}$ & 97 & & & & \\
\hline $\begin{array}{l}\text { Aid to quit smoking } \\
\text { cigarettes }\end{array}$ & 34 & 35.1 & 35.9 & 33.3 & \multirow{5}{*}{0.65} \\
\hline $\begin{array}{l}\text { Healthier than } \\
\text { cigarettes }\end{array}$ & 32 & 32.9 & 34.4 & 30.3 & \\
\hline It is enjoyable & 20 & 20.6 & 21.9 & 18.2 & \\
\hline Cheaper than cigarettes & 5 & 5.2 & 3.1 & 9.1 & \\
\hline Other & 6 & 6.2 & 4.7 & 9.1 & \\
\hline $\begin{array}{l}\text { Intention to reduce } \\
\text { nicotine }\end{array}$ & 99 & & & & \\
\hline Yes & 60 & 60.6 & 70.4 & 48.9 & \multirow{3}{*}{0.004} \\
\hline No & 30 & 30.3 & 16.7 & 46.7 & \\
\hline Don't know & 9 & 9.1 & 12.9 & 4.4 & \\
\hline $\begin{array}{l}\text { Intention to quit } \\
\text { vaping }\end{array}$ & 99 & & & & \\
\hline Yes & 48 & 48.5 & 47.0 & 51.5 & \multirow{3}{*}{0.11} \\
\hline No & 27 & 27.3 & 33.3 & 15.2 & \\
\hline Don't know & 24 & 24.2 & 19.7 & 33.3 & \\
\hline
\end{tabular}

a Participants were recruited from Maryland from December 2015 - October 2017.
5 to 240 (median: 32.5 ) $\mathrm{mL} /$ week, with women consuming less per week than men, and individuals with a higher level of education consuming less per week than those with a lower level of education. The median (range) nicotine concentration was $3.0(0-24)$ $\mathrm{mg} / \mathrm{mL}$. The median (IQR) number of puffs per day was 200 (90-360) puffs, with each puff estimated to last an average of 4 (SD: 2) seconds. Older aged participants preferred higher nicotine concentrations in e-liquid and fewer puffs/day. Seconds/puff was not associated with demographic characteristics.

The primary reasons for vaping were to quit smoking cigarettes (35\%), to use as a healthier alternative to cigarettes (33\%), and because it is enjoyable $(21 \%)$ (Table 4$)$. The intention to reduce nicotine concentration of e-liquid was lower in women $(48.9 \%)$ compared to men (70.4\%). Overall, a little less than $50 \%$ of e-cigarette users reported an intention to quit vaping.

\section{Self-reported health status and home rules with tobacco/e-cigarette use}

Regarding general health characteristics, e-cigarette users were more likely to report symptoms of wheezing and whistling in the chest (15\% vs $2 \%$, $\mathrm{p}=0.02)$ as well as having hypertension $(22 \%$ vs $4 \%$, $\mathrm{p}=0.007$ ) than non-users (Supplementary file, Table S1). After running additional analyses adjusting for age, sex, and previous smoking status, this was not statistically significant. Twenty-seven of the e-cigarette users reported sensory and respiratory symptoms (sore throat, runny nose, bringing up phlegm, and coughing) occurring with e-cigarette use. While there was no difference with banning cigarette smoking inside the home between users (64\%) and non-users $(62 \%)$, most e-cigarette users $(89 \%)$ had no rules on banning vaping indoors, compared to nonusers (50\%) (Supplementary file, Table S2).

\section{DISCUSSION}

In our study sample from Maryland between 2015 and 2017, the majority of daily exclusive e-cigarette users were men, White, former smokers, and used open system devices (MODs/tanks). This is consistent with data from the nationally representative Population Assessment of Tobacco and Health (PATH) study (Waves 1 and 2), where exclusive use of e-cigarettes was more prevalent among non-Hispanic Whites 
compared to non-Hispanic Blacks and Hispanics ${ }^{10}$, and those who reported using open-system devices were more likely to report daily use compared to those who did not use this type of device ${ }^{11}$. Prior studies have also found ever use of e-cigarettes to be higher among men than women ${ }^{12,13}$. This study differs from prior e-cigarette research as it focuses on daily exclusive e-cigarette users, their behaviors and device preferences that may influence toxic exposures from daily e-cigarette use, and the differences in health characteristics and house rules of tobacco use between users and non-users.

According to the reported behaviors of e-cigarette users, close to a third of our participants first vape within 5 minutes of waking in the morning and more than half vape throughout the day, indicating a high level of nicotine dependence. Older aged individuals vaped e-liquids of higher nicotine concentrations but reported lower total puffs per day. These findings are consistent with a study of nicotine dependence and consumption among e-cigarette users mostly based in the United Kingdom, Australia, Finland, Ireland and the United States, which found that older users preferred a high nicotine-concentration and low power style of vaping ${ }^{14}$. With a higher level of nicotine, fewer puffs would be necessary for nicotine delivery.

E-cigarette users in our study changed their coils on average 3 times per month. No other study has reported on the frequency of coil change. This is an important behavior, as studies ${ }^{15}$ have found elements from coil alloys such as nickel and chromium in the aerosol that is inhaled by the user, and an increased frequency of coil change has been associated with higher metal biomarker levels ${ }^{15}$. The most frequently reported coil types in this study (Kanthal, stainless steel, and Nichrome) contain chromium (Cr) and/or nickel (Ni). Our group has found that the levels of these two metals in the aerosol correlate with metal levels in urine or saliva from the same participants ${ }^{15}$. We also found that metal levels are, in general, higher in the aerosol than in the original liquid ${ }^{6}$, supporting the finding that metal exposure from e-cigarette devices is likely derived, at least in part, from the heating coils. This is concerning as inhalation of nickel and chromium is known to cause airway irritation and obstruction, as well as lung, nasal, and sinus cancer ${ }^{16}$.

Participants reported using e-cigarettes primarily as an aid to quit smoking (35\%) and as a healthier alternative to combustible cigarettes (32\%). This is consistent with current established adult e-cigarette users from Wave 1 of the PATH study (2013-2014) who also reported using e-cigarettes as an alternative to cigarettes ${ }^{17}$. Interestingly, women in our study were less inclined to reduce their nicotine e-liquid concentrations compared to men. This is consistent with smoking cessation studies that have found that, compared to men, women have higher nicotine dependence and are less successful in quitting tobacco use ${ }^{18,19}$. Alternatively, it could be due to a lower nicotine flux, which is the nicotine emitted per puff-second $(\mathrm{mg} / \mathrm{s})$ that is not only determined by the e-liquid concentration used but also by the device characteristics (i.e. voltage or power settings) and the use puff topography (i.e. seconds/puff, puffs/ day $)^{20}$. Compared to men, women in our study had the same preferred mean e-liquid nicotine concentration $(5.3 \mathrm{mg} / \mathrm{mL})$. However, they vaped their devices at a lower voltage (mean difference: $0.54 \mathrm{~V}, 95 \%$ CI: -1.04, -0.03) and drew fewer puffs/day (mean difference: $-132 ; 95 \%$ CI: $-49.9,-0.25)$, indicating that the amount of nicotine they receive is likely lower than in men.

Overall, $48.5 \%$ of our study population intended to quit vaping altogether, which is lower than the findings from the PATH study (Wave 3: 2015-2016) where nearly two-thirds of e-cigarette users (62.4\%) planned to quit e-cigarettes ${ }^{21}$. This PATH study sample, however, includes current e-cigarettes users who vape daily and also those who vape some days, which may account for this difference, and while $62.4 \%$ of users in the PATH study reported plans to quit, most of these users' timeframe for quitting is a year or longer ( $8 \%$ plan to quit within the next 7 days, $7.7 \%$ in the next month, $13 \%$ in the next 6 months, $33 \%$ in the next year, $38 \%$ longer than that). Moreover, more than $25 \%$ of users in the PATH study reported unsuccessful e-cigarette quit attempts in the past year, indicating that quitting e-cigarette use may be a challenge, similar to quitting traditional cigarettes ${ }^{21}$.

Compared to non-users, e-cigarette users were more likely to report symptoms of wheezing and whistling in the chest as well as having hypertension, although after adjusting for sex, age, and former smoking status, this was not statistically significant. An assessment of Wave 2 of the PATH study also found 
an increased risk of wheezing and related respiratory symptoms among current e-cigarette users compared to non-users, but a lower risk when compared to current smokers or dual users ${ }^{22}$, which are groups we did not recruit in this study.

While our participants reported no difference in house rules regarding banning smoking cigarettes indoors, e-cigarette users were less likely to have rules in place for vaping indoors compared to non-users. This is concerning for both users and bystanders as exposure to e-cigarette aerosol is not without risks since several components have demonstrated toxicological health effects. For example, exposure to propylene glycol, a humectant in e-liquids, has been shown to decrease membrane fluidity in airway epithelia and increase mucin expression after vaping ${ }^{23}$. Moreover, exposure to nicotine delivered by e-cigarettes has been found to increase arterial stiffness and affect microcirculation ${ }^{24}$, which indicates that e-cigarettes use may be a risk factor for cardiovascular disease.

Furthermore, participants in our study reported using their devices daily, throughout the day, at a few hundred puffs per day, with behaviors that may further compound exposures. For instance, in Wave 1 of the PATH study, 'daily' e-cigarette users had significantly higher urinary metal levels $(\mathrm{Pb}$ and $\mathrm{Sr}$ ) compared to 'some days' users ${ }^{25}$. This may be, in part, due to the increased e-liquid consumption ${ }^{15}$ and leaching of metals into the liquid and aerosol through contact with the coil ${ }^{6}$. In our study, men were more likely to vape at a higher voltage and consume more e-liquid per week than women. This higher intensity of use among men has also been reported in other studies ${ }^{12,13}$. There is also evidence that increasing the voltage, and thereby, power, influences reactive oxygen species (ROS) formation ${ }^{26}$, and shifts the particle mass distribution towards smaller particles and increases the respirable fraction of aerosol to enter ciliated airways ${ }^{27}$. Increasing power has also been associated with higher levels of degradation products released into the aerosol, such as aldehydes, acrolein, diacetyl, and formaldehyde ${ }^{28}$. Users in our study vaped at an average voltage of $4.21 \mathrm{~V}$ (range: $2.12-12.5$ ) and an average of 365 puffs/day (range: $15-1000$ ). This is concerning as Logue et al. ${ }^{29}$ calculated that users with a vaping regimen of 250 puffs/day using a tank device at voltages from 3.8 to $4.8 \mathrm{~V}$ can inhale up to 49 $\mathrm{mg}$ /day formaldehyde, up to $10 \mathrm{mg}$ /day acrolein and up to $0.5 \mathrm{mg} /$ day diacetyl, levels that exceeded US occupational limits ${ }^{29}$, suggesting that concentrations of these degradation products are relevant to health.

\section{Limitations}

This study has several limitations. First, our study could be affected by selection bias due to convenience sampling. While both groups (e-cigarette users and non-users) were matched according to sex, age, and race, the majority of non-users $(90 \%)$ had a higher level of education and were current students compared to e-cigarette users (59\%). Second, e-cigarette use behaviors (such as e-liquid consumed/week, number of puffs/day, average seconds/puff, time to first vape after waking, etc.) are based on self-report and it is possible that participants could have displayed recall or social desirability bias. Third, as we only recruited participants aged $\geq 18$ years, and since the use of 4th generation PODs (Juul, Suorin, etc.) rose in popularity towards the tail-end of our recruitment in 2017, we are likely missing an important population of e-cigarette users, particularly among youth of middle school and high school age.

\section{CONCLUSIONS}

This study provides important information regarding behaviors of daily exclusive e-cigarette users. Most daily e-cigarette users were male, White, former smokers, owned an average of 2 open-system devices, and vaped an average of 365 puffs/day throughout the day. Men were more likely to vape at a higher voltage than women. Men and users with lower education consumed more e-liquid/week than their respective counterparts, suggesting a higher exposure to toxic compounds. Women expressed less desire to lower nicotine levels in their e-liquid compared to men. With daily use and no intention to quit vaping, e-cigarette users may be at risk for long-term health effects from exposures to e-cigarette by-products. Future research, in particular nationwide surveys, should document the practices of daily e-cigarette users, particularly related to the coil, power, and nicotine used in e-liquid, as the levels of both organic and inorganic compounds with known toxicological health effects are contingent on these parameters. Given the heterogeneity of e-cigarettes in the market and the ability of users to modify device characteristics, research studies looking at e-cigarette constituents and health effects 
should include a comprehensive characterization of e-cigarette use behaviors and device characteristics.

\section{REFERENCES}

1. Gentzke AS, Creamer M, Cullen KA, et al. Vital Signs: Tobacco Product Use among Middle and High School Students - United States, 2011-2018. MMWR Morb Mortal Wkly Rep. 2019;68(6):157-164. doi:10.15585/ mmwr.mm6806e1

2. Chen C, Zhuang YL, Zhu SH. E-Cigarette Design Preference and Smoking Cessation: A U.S. Population Study. Am J Prev Med. 2016;51(3):356-363. doi:10.1016/j.amepre.2016.02.002

3. Walley SC, Jenssen BP, Section on Tobacco Control. Electronic Nicotine Delivery Systems. Pediatrics. 2015;136(5):1018-1026. doi:10.1542/peds.2015-3222

4. Bhatnagar A, Whitsel LP, Ribisl KM, et al. Electronic Cigarettes: A Policy Statement from the American Heart Association. Circulation. 2014;130(16):1418-1436. doi:10.1161/cir.0000000000000107

5. Delnevo C, Giovenco DP, Hrywna M. Rapid Proliferation of Illegal Pod-Mod Disposable E-Cigarettes. Tob Control. 2020. doi:10.1136/tobaccocontrol-2019-055485

6. Olmedo P, Goessler W, Tanda S, et al. Metal Concentrations in E-Cigarette Liquid and Aerosol Samples: The Contribution of Metallic Coils. Environ Health Perspect. 2018;126(2):027010. doi:10.1289/ ehp2175

7. Sharapova SR, Singh T, Agaku IT, Kennedy SM, King BA. Patterns of E-Cigarette Use Frequency-National Adult Tobacco Survey, 2012-2014. Am J Prev Med. 2018;54(2):284-288. doi:10.1016/j.amepre.2017.09.015

8. Heatherton TF, Kozlowski LT, Frecker RC, Fagerstrom KO. The Fagerstrom Test for Nicotine Dependence: A Revision of the Fagerstrom Tolerance Questionnaire. Br J Addict. 1991;86(9):1119-1127. doi:10.1111/j.1360-0443.1991.tb01879.x

9. Menzies D, Nair A, Williamson PA, et al. Respiratory Symptoms, Pulmonary Function, and Markers of Inflammation among Bar Workers before and after a Legislative Ban on Smoking in Public Places. JAMA. 2006;296(14):1742-1748. doi:10.1001/jama.296.14.1742

10. Harlow AF, Stokes A, Brooks DR. Socioeconomic and Racial/Ethnic Differences in E-Cigarette Uptake among Cigarette Smokers: Longitudinal Analysis of the Population Assessment of Tobacco and Health (Path) Study. Nicotine Tob Res. 2019;21(10):1385-1393. doi:10.1093/ntr/nty141

11. Coleman BN, Rostron B, Johnson SE, et al. Electronic Cigarette Use among Us Adults in the Population Assessment of Tobacco and Health (Path) Study, 20132014. Tob Control. 2017;26(e2):e117-e126. doi:10.1136/ tobaccocontrol-2016-053462

12. Pineiro B, Correa JB, Simmons VN, et al. Gender
Differences in Use and Expectancies of E-Cigarettes: Online Survey Results. Addict Behav. 2016;52:91-97. doi:10.1016/j.addbeh.2015.09.006

13. Schoenborn CA, Gindi RM. Electronic Cigarette Use among Adults: United States, 2014. NCHS Data Brief. 2015(217):1-8. PMID:26555932

14. Browne M, Todd DG. Then and Now: Consumption and Dependence in E-Cigarette Users Who Formerly Smoked Cigarettes. Addict Behav. 2018;76:113-121. doi:10.1016/j.addbeh.2017.07.034

15. Aherrera A, Olmedo P, Grau-Perez M, et al. The Association of E-Cigarette Use with Exposure to Nickel and Chromium: A Preliminary Study of NonInvasive Biomarkers. Environ Res. 2017;159:313-320. doi:10.1016/j.envres.2017.08.014

16. Agency for Toxic Substances and Disease Registry. Chromium Toxicity: What Are the Physiologic Effects of Chromium Exposure? https://www.atsdr.cdc.gov/csem/ csem.asp?csem $=10 \&$ po $=10$. Published 2008. Accessed November 30, 2019.

17. Nicksic NE, Snell LM, Barnes AJ. Reasons to Use E-Cigarettes among Adults and Youth in the Population Assessment of Tobacco and Health (Path) Study. Addict Behav. 2019;93:93-99. doi:10.1016/j. addbeh.2019.01.037

18. Komiyama M, Yamakage H, Satoh-Asahara N, et al. Sex Differences in Nicotine Dependency and Depressive Tendency among Smokers. Psychiatry Res. 2018;267:154159. doi:10.1016/j.psychres.2018.06.010

19. Garey L, Peraza N, Smit T, et al. Sex Differences in Smoking Constructs and Abstinence: The Explanatory Role of Smoking Outcome Expectancies. Psychol Addict Behav. 2018;32(6):660-669. doi:10.1037/adb0000391

20. Shihadeh A, Eissenberg T. Electronic Cigarette Effectiveness and Abuse Liability: Predicting and Regulating Nicotine Flux. Nicotine Tob Res. 2015;17(2):158-162. doi:10.1093/ntr/ntu175

21. Rosen RL, Steinberg ML. Interest in Quitting E-Cigarettes among Adults in the United States. Nicotine Tob Res. 2020;22(5):857-858. doi:10.1093/ntr/ntz062

22. Li D, Sundar IK, McIntosh S, et al. Association of Smoking and Electronic Cigarette Use with Wheezing and Related Respiratory Symptoms in Adults: Cross-Sectional Results from the Population Assessment of Tobacco and Health (Path) Study, Wave 2. Tob Control. 2020;29:140-147. doi:10.1136/tobaccocontrol-2018-054694

23. Reidel B, Radicioni G, Clapp PW, et al. E-Cigarette Use Causes a Unique Innate Immune Response in the Lung, Involving Increased Neutrophilic Activation and Altered Mucin Secretion. Am J Respir Crit Care Med. 2018;197(4):492-501. doi:10.1164/rccm.201708-1590oc

24. Chaumont M, de Becker B, Zaher W, et al. Differential Effects of E-Cigarette on Microvascular Endothelial Function, Arterial Stiffness and Oxidative Stress: A Randomized Crossover Trial. Sci Rep. 2018;8(1). 
doi:10.1038/s41598-018-28723-0

25. Goniewicz ML, Smith DM, Edwards KC, et al. Comparison of Nicotine and Toxicant Exposure in Users of Electronic Cigarettes and Combustible Cigarettes. JAMA Netw Open. 2018;1(8):e185937. doi:10.1001/ jamanetworkopen.2018.5937

26. Zhao J, Zhang Y, Sisler JD, et al. Assessment of Reactive Oxygen Species Generated by Electronic Cigarettes Using Acellular and Cellular Approaches. J Hazard Mater. 2018;344:549-557. doi:10.1016/j.jhazmat.2017.10.057

27. Floyd EL, Queimado L, Wang J, Regens JL, Johnson DL. Electronic Cigarette Power Affects Count Concentration and Particle Size Distribution of Vaping Aerosol. PLoS One. 2018;13(12):e0210147. doi:10.1371/journal. pone. 0210147

28. Salamanca JC, Meehan-Atrash J, Vreeke S, Escobedo JO, Peyton DH, Strongin RM. E-Cigarettes Can Emit Formaldehyde at High Levels under Conditions That Have Been Reported to Be Non-Averse to Users. Sci Rep. 2018;8(1):7559. doi:10.1038/s41598-018-25907-6

29. Logue JM, Sleiman M, Montesinos VN, et al. Emissions from Electronic Cigarettes: Assessing Vapers' Intake of Toxic Compounds, Secondhand Exposures, and the Associated Health Impacts. Environ Sci Technol. 2017;51(16):9271-9279. doi:10.1021/acs.est.7b00710
ACKNOWLEDGEMENTS

We express our gratitude to Walla Alfaraj, Maria Andrade, Patryk Krzesaj, and Kiana Rodjers, for aiding in participant recruitment as well as to those who participated in this study.

\section{CONFLICTS OF INTEREST}

The authors have completed and submitted the ICMJE Form for Disclosure of Potential Conflicts of Interest and none was reported.

\section{FUNDING}

This work was supported by the Maryland Cigarette Restitution Fund (Proposal Number: PHPA-G2034), Alfonso Martin Escudero Foundation to PO, and the American Heart Association Tobacco Regulation and Addiction Center (Proposal Number: 1P50HL120163) to AA. Research reported in this publication was also supported by the National Institute of Environmental Health Sciences of the National Institutes of Health under Award Number R01ES030025. The content is solely the responsibility of the authors and does not necessarily represent the official views of the National Institutes of Health. None of the funding sources played any role in the design of the study and collection, analysis, and interpretation of data, or in writing the manuscript.

\section{AUTHORS' CONTRIBUTIONS}

AMR and ANA developed the idea for this research. AA, AAr, SJ, PO and $\mathrm{RC}$ conducted recruitment, data collection, and laboratory analysis. $\mathrm{AA}$ and $\mathrm{AAr}$ wrote the first draft of the article. AA and AAr conducted the data analysis and developed the tables. Pre-submission review and editing were completed by AA, AAr, SJ, PO, RC, JEC, ANA and AMR. AMR is the guarantor.

PROVENANCE AND PEER REVIEW

Not commissioned; externally peer reviewed. 